Georgian Mathematical Journal

Volume 12 (2005), Number 1, 11-13

\title{
THE NON-VANISHING OF FIRST COHOMOLOGY GROUPS FOR CERTAIN INFINITE-DIMENSIONAL COMPLEX MANIFOLDS
}

\author{
E. BALLICO
}

\begin{abstract}
Here, using the ideas of an old paper by S. Dineen (1976), we give large classes of pairs $(X, E)$ such that $X$ is an infinite-dimensional complex space very far from a Banach manifold, $E$ is a holomorphic vector bundle on $X$ and $H^{1}(X, E)$ is infinite-dimensional.
\end{abstract}

2000 Mathematics Subject Classification: 32L05, 32K05.

Key words and phrases: Infinite-dimensional complex manifold, first Cousin problem, additive Cousin problem, non-vanishing of cohomology.

Let $V$ be a complex locally convex and Hausdorff topological vector space. A sequence $\left\{x_{n}\right\}_{n \geq 1} \subset V$ is called a nontrivial very strongly convergent sequence if the sequence $\left\{\lambda_{n} x_{n}\right\}_{n \geq 1}$ converges to $0 \in V$ for all $\lambda_{n} \in \mathbf{C}$ and $x_{n} \neq 0$ for all $n$. For instance, if $V=\mathbf{C}^{\mathbf{N}}$, then the sequence $(1,0,0, \ldots),(0,1,0, \ldots), \ldots$ is a nontrivial very strongly convergent sequence. By [4], Th. 2.6.13, a Fréchet space contains $\mathbf{C}^{\mathbf{N}}$ if and only if it has no continuous norm. Hence a Fréchet space has a continuous norm if and only if it has a nontrivial strongly convergent sequence. The aim of this short note is to give the following generalization of [1], Prop. 1; we will mostly use the ideas contained in [1].

Theorem. Let $V$ be a complex locally convex and Hausdorff topological vector space which admits a nontrivial very strongly convergent sequence $\left\{x_{n}\right\}_{n \geq 1}$ and $X$ a reduced and locally integral complex space equipped with a holomorphic map $f: X \rightarrow V$ with the following property:

$(\alpha)$ for every $P \in X$ there are an open neighborhood $A$ of $P$ in $X$ and an open neighborhood $B$ of $f(P)$ in $V$ such that $f \mid A$ is a closed embedding of $A$ into $B$ and the analytic set $f(A)$ is the zero-locus of finitely many holomorphic functions on $B$.

Let $E$ be a holomorphic vector bundle on $X$ such that $H^{0}(X, E) \neq 0$. Then $H^{1}(X, E)$ is an infinite-dimensional $\mathbf{C}$-vector space.

In the statement of Theorem we allow the case in which the fibers of $E$ are infinite-dimensional complex topological vector spaces.

Remark. We use the notation introduced in the statement of Theorem. We also assume that $X$ is integral. Let $g$ be a meromorphic function on $X$. Then $g$ depends locally only on finitely many variables $x_{n}$ in the following sense: for every $P \in X$ we take $A$ and $B$ as in the statement of Theorem. Consider $(g \mid A) \otimes\left(f \mid f^{-1}(f(A))\right.$ as a meromorphic function $g^{\prime}$ on $f(A)$. Then there are 
a neighborhood $D$ of $f(P)$ in $B$ and a meromorphic function $g^{\prime \prime}$ on $B^{\prime}$ such that $g^{\prime \prime} \mid f(A) \cap D=g^{\prime}$. By [1], Lemma 1, there is an integer $N>0$ such that $\partial g^{\prime \prime} / \partial x_{n} \equiv 0$ for every $n \geq N$.

Proof of Theorem. By assumption, $X$ is locally finitely determined in the sense of [3] and hence the set $X_{\text {reg }}$ is an open dense subset of $X$. Fix $s \in H^{0}(X, E)$, $s \neq 0$. Hence $s$ does not vanish at each point of a dense open subset of $X$ whose complement is an analytic subset of $X$. In particular, $s$ does not vanish in an open and dense subset of $X_{\text {reg. }}$. Fix $P \in X_{\text {reg }}$ such that $s(P) \neq 0$. By assumption $(\alpha)$, near $P f(X)$ is a complex submanifold of $V$ with finite codimension and hence its tangent space $T_{P} f(X)$ at $P$ is a finite codimensional affine linear subspace of $V$. Deleting finitely many members of the sequence $\left\{x_{n}\right\}_{n \geq 1}$, we may assume that the vector space $T_{P} f(X)-P$ contains each $x_{n}$. Since $X$ is locally integral, to prove the theorem it is sufficient to prove it with the additional assumption that $X$ is integral. Let $\mathcal{M}_{X}$ be the sheaf of meromorphic functions on $X$; for the general theory of $\mathcal{M}_{X}$ when $X$ is not smooth, see [2]. Since $\mathcal{O}_{X}$ is a subsheaf of $\mathcal{M}_{X}$, there is an exact sequence

$$
0 \rightarrow \mathcal{O}_{X} \rightarrow \mathcal{M}_{X} \rightarrow \mathcal{M}_{X} / \mathcal{O}_{X} \rightarrow 0
$$

This is the set-up of the so-called Cousin's first problem or additive Cousin problem. Since $E$ is locally free, tensoring (1) with $E$ we obtain an exact sequence of $\mathcal{O}_{X}$-sheaves

$$
0 \rightarrow E \rightarrow \mathcal{M}_{X} \otimes_{\mathcal{O}_{X}} E \rightarrow\left(\mathcal{M}_{X} / \mathcal{O}_{X}\right) \otimes_{\mathcal{O}_{X}} E \rightarrow 0
$$

Thus to prove Theorem it is sufficient to show that the linear map

$$
\rho: H^{0}\left(X, \mathcal{M}_{X} \otimes_{\mathcal{O}_{X}} E\right) \rightarrow H^{0}\left(X,\left(\mathcal{M}_{X} / \mathcal{O}_{X}\right) \otimes_{\mathcal{O}_{X}} E\right)
$$

has the infinite-dimensional cokernel. First, we will check that $\rho$ is not surjective. For every integer $n \geq 1$, let $A_{n} \subset V$ be the linear span of $\left\{x_{1}, \ldots, x_{n}\right\}$. Since $A_{n}$ is finite-dimensional, it has a topological supplement in $V$ by HahnBanach theorem. Construct inductively a decreasing sequence of closed subspaces $F_{n}, n \geq 1$, of $V$ such that $F_{n}$ is a topological supplement of $A_{n}$. Set $U_{2}:=\left\{c x_{1}+w: c \in \mathbf{C}, \operatorname{Im}(c)<11 / 4\right.$ and $\left.w \in F_{1}\right\}$. For each $n>2$ set $U_{n}:=\left\{c x_{1}+w: c \in \mathbf{C}, n-3 / 4<\operatorname{Im}(c)<n+3 / 4\right.$ and $\left.v \in F_{1}\right\}$. Set $X_{n}:=f^{-1}\left(U_{n}\right)$. We may define the elements $\alpha_{i} \in V^{\prime}, i \geq 1$ by the relations $v=\sum_{i=1}^{n} \alpha_{i}(v) x_{i}+v_{n}$ with $v_{n} \in F_{n}$ for any $v \in V$. For $n \geq 2$ and any $x \in X$ set $f_{n}(x):=s(x) \alpha_{n}(f(x)) /\left(\alpha_{1}(f(x))-i n\right)$. As in [1], using Remark we get that this definition gives a nontrivial element of $\operatorname{Coker}(\rho)$. Now we will check that $H^{1}(X, E)$ is infinite-dimensional. Assume $\operatorname{dim}\left(H^{1}(X, E)\right)=k<+\infty$ and fix $z \in V^{\prime} \backslash\{0\}$ such that the set $\left\{z\left(x_{n}\right)\right\}_{n \geq 1} \subset \mathbf{C}$ contains at least $k+1$ elements. For every $u \in H^{0}\left(V, \mathcal{O}_{V}\right), f^{*}(u) \in H^{0}\left(X, \mathcal{O}_{X}\right)$ and hence the multiplication by $f^{*}(u)$ induces a linear map $f^{*}(u) \times: H^{1}(X, E) \rightarrow H^{1}(X, E)$. For every polynomial $q \in \mathbf{C}[x]$ we have $q\left(f^{*}(u) \times\right)=\left(f^{*}(q(u))\right) \times$. Hence by Hamilton-Cayley there is $q \in \mathbf{C}[x]$ such that $q \neq 0, \operatorname{deg}(q) \leq k$ and $f^{*}(q(z)) \times=0$. Instead of the section $s$ of $E$ use the section $f^{*}(q(z)) s$ to obtain a contradiction. 


\section{ACKNOWLEDGEMENT}

The author was partially supported by MIUR and GNSAGA of INdAM (Italy).

\section{REFERENCES}

1. S. DineEn, Cousin's first problem on certain locally convex topological vector spaces. An. Acad. Brasil. Ci. 48(1976), No. 1, 11-12.

2. S. L. Kleiman, Misconceptions about $K_{x}$. Enseign. Math. (2) 25(1979), No. 3-4, 203206 (1980).

3. P. MAZet, Analytic sets in locally convex spaces. North-Holland Mathematics Studies, 89. Notas de Matemática [Mathematical Notes], 93. North-Holland Publishing Co., Amsterdam, 1984.

4. P. PÉrez Carreras and J. Bonet, Barrelled locally convex spaces. North-Holland Mathematics Studies, 131. Notas de Matemática [Mathematical Notes], 113. NorthHolland Publishing Co., Amsterdam, 1987.

(Received 6.01.2004)

Author's address:

Dept. of Mathematics

University of Trento

38050 Povo (TN), Italy

E-mail: ballico@science.unitn.it 